Abanico Veterinario. Enero-Diciembre 2020; 10(1):1-11. http://dx.doi.org/10.21929/abavet2020.1 Artículo Original. Recibido: 25/02/2019. Aceptado: 16/09/2019. Publicado: 06/01/2020.

\title{
Efecto antibacteriano del extracto metanólico de Salix babylonica sobre bacterias de importancia en salud pública
}

\author{
Antibacterial effect of the methanol extract of Salix babylonica against important \\ bacteria in public Health
}

\section{González-Alamilla Eddy'(D), Rivas-Jacobo Marco'1D, Sosa-Gutiérrez Carolina2 ${ }^{1 D}$, Delgadillo-Ruiz Lucía ${ }^{3}$, Valladares-Carranza Benjamín ${ }^{4}$, Rosenfeld-Miranda Carla $^{5}$ iD, Zaragoza-Bastida Adrián ${ }^{2^{*}}$, Rivero-Pérez Nallely ${ }^{2^{*}}$}

${ }^{1}$ Universidad Autónoma de San Luis Potosí, Facultad de Agronomía y Veterinaria. ²Universidad Autónoma del Estado de Hidalgo, Instituto de Ciencias Agropecuarias, Área Académica de Medicina Veterinaria y Zootecnia. ${ }^{3}$ Unidad Académica de Ciencias Biológicas, Universidad Autónoma de Zacatecas. Zacatecas, México. ${ }^{4}$ Universidad Autónoma del Estado de México, Facultad de Medicina Veterinaria y Zootecnia, ${ }^{5}$ Facultad de Ciencias Veterinarias, Universidad Austral de Chile, Isla Teja s/n, Casilla 567, Valdivia, Chile *Autor responsable y de correspondencia: Adrián Zaragoza-Bastida, Nallely Rivero-Perez. Rancho universitario Av. Universidad km. 1, Ex Hacienda de Aquetzalpa, Apartado Postal No. 32, Tulancingo de Bravo, Hidalgo, México. eddynglez24@gmail.com, marco.rivas@uaslp.mx, carolina_sosa@uaeh.edu.mx, delgadillolucia@gmail.com, crosenfe@uach.cl, adrian_zaragoza@uaeh.edu.mx, nallely_rivero@uaeh.edu.mx.

\section{RESUMEN}

El uso excesivo de antimicrobianos ha generado resistencia de los microorganismos a estos, se han buscado alternativas que sean eficaces para el tratamiento de enfermedades producidas por microorganismos resistentes o multirresistentes a antibióticos, dentro de estas alternativas están las plantas, las cuales por su contenido de compuesto secundarios presentan actividad antibacteriana. El objetivo del presente estudio fue caracterizar y determinar la actividad antibacteriana del extracto metanólico de Salix babylonica (SB) sobre bacterias de importancia en salud pública. Para la obtención del extracto se utilizó la técnica de maceración, se realizó una caracterización química cualitativa y cuantitativa por cromatografía de gases. Para determinar la actividad antibacteriana, se determinó la Concentración Mínima Inhibitoria (CMI) y la Concentración Mínima Bactericida (CMB) y la caracterización del extracto permitió identificar compuestos fenólicos, cumarinas, lactonas, flavonoles, quinonas, saponinas, triterpenos y compuestos esteroidales, además de Timol $(0.5319 \mathrm{mg} / \mathrm{mL})$ y Carvacrol $(0.4158 \mathrm{mg} / \mathrm{ml})$. Con respecto a la actividad antibacteriana la mejor actividad se presentó contra Bacillus. subtillis (CMl: $12.5 \mathrm{mg} / \mathrm{mL}$ y CMB: $25 \mathrm{mg} / \mathrm{mL}$ ), Listeria. monocytogenes y Staphylococcus. aureus (CMI: $25 \mathrm{mg} / \mathrm{mL}$ y CMB: $50 \mathrm{mg} / \mathrm{mL}$ ). Se concluye que el extracto metanólico de SB puede ser una alternativa para el tratamiento de enfermedades producidas por bacterias resistentes o multirresistentes a antibióticos.

Palabras clave: Salix babylonica, caracterización, efecto antibacteriano.

\begin{abstract}
The excessive use of antibiotics, has generated resistance of microorganisms to these, have been searched effectives alternatives for treating diseases caused by resistant or multiresistant microorganism, within of these alternatives are plants, which by its content of secondary compounds have antibacterial activity. The aim on the present experiment was characterize and determine the antibacterial activity of methanolic extract of Salix babylonica (SB) against important bacteria in public health. To obtain extract, the maceration technique was used, qualitative and quantitative (gas chromatography) chemical characterization was carried. For antibacterial activity, the Minimum Inhibitory Concentration (MIC) and Minimum Bactericidal Concentration (MBC) was determined, the characterization of the extract allowed the identification of
\end{abstract}


phenolic compounds, coumarins, lactones, flavonols, quinones, saponins, triterpenes and steroidal compounds, also Thymol $(0.5319 \mathrm{mg} / \mathrm{mL})$ and Carvacrol $(0.4158 \mathrm{mg} / \mathrm{mL})$. The extract showed the best activity against Bacillus. subtilis (MIC: $12.5 \mathrm{mg} / \mathrm{mL}$ and WBC: $25 \mathrm{mg} / \mathrm{mL}$ ), Listeria. monocytogenes and Staphylococcus. aureus (MIC: $25 \mathrm{mg} / \mathrm{mL}$ and MBC: $50 \mathrm{mg} / \mathrm{mL}$ ). It is concluded that the methanolic extract of SB can be an alternative for the treatment of diseases produced by resistant or multiresistant bacteria to antibiotics.

Keywords: Salix babylonica, characterization, antibacterial effect.

\section{INTRODUCCIÓN}

Las enfermedades infecciosas provocadas por microorganismos, han sido unas de las causas más importantes de muerte en la humanidad (Lozano et al., 2012). Los agentes bacterianos incluyendo Staphylococcus aureus, Escherichia coli, Pseudonomas aeruginosa, Bacillus subtilis y Proteus vulgaris han provocado enfermedades infecciosas importantes dentro de la salud pública (Khan et al., 2013).

La introducción de agentes antimicrobianos en la medicina, ha sido una de las intervenciones más importantes para controlar y disminuir la prevalencia de las enfermedades infecciosas (Alós, 2015); sin embargo, una amenaza creciente en los últimos años que ha disminuido la eficacia de estos fármacos, es la resistencia bacteriana a los antibióticos; generada debido a que los microorganismos han adquirido la capacidad para evitar que un antimicrobiano actúe contra él. Como resultado, los tratamientos de elección se vuelven ineficaces, las infecciones persisten y pueden extenderse a otros individuos (WHO, 2017).

En la mayoría de las poblaciones de diferentes países en desarrollo, la humanidad ha usado plantas para tratar enfermedades infecciosas comunes, que podrían ser una alternativa potencial para producir nuevos fármacos de gran beneficio a la salud (Renisheya et al., 2011; Khan et al., 2013).

Una de las plantas considerada importantes para el estudio de sus propiedades fotoquímicas es Salix babylonica, conocido comúnmente como Sauce llorón. Esta especie pertenece al género Salix de la familia Salicáceas, Salix babylonica es una de las especies más conocidas dentro de los sauces, distribuido en algunas zonas de Asia, Europa y América; utilizada comúnmente como planta ornamental y medicinal (Wahab et al., 2018).

Existen reportes en los que se evidencian las propiedades farmacológicas asociadas a la evaluación de extractos de hojas, corteza y tallos; obtenidos a partir de Salix babylonica. Dentro de las propiedades fitoquímicas atribuibles a Salix babylonica, se encuentran: actividad antihelmíntica, antiséptica, antiartrítica, astringente, analgésica, anticancerígena, antipirética, antimalaria, antioxidante, antimicótica, antihelmíntica y antibacteriana; estas propiedades su asocian a su contenido de compuestos secundarios como fenólicos totales, flavonoides, terpenos y lignanos (Sulaiman et al., 2013; Wahab et al., 2018). 
Con base en los planteamientos anteriormente mencionados, el objetivo del presente trabajo de investigación fue caracterizar y determinar la actividad antibacteriana del extracto metanólico de Salix babylonica, sobre bacterias de importancia en salud pública.

\section{MATERIAL Y MÉTODOS}

\section{Obtención del extracto}

Para la obtención del extracto se recolectó aproximadamente $1 \mathrm{~kg}$ de material vegetal de Salix babylonica en diferentes etapas fenológicas, éstas fueron colectadas en el municipio de Tulancingo, Hidalgo. La parte aérea recolectada de Salix babylonica se llevó a secado a sombra a temperatura ambiente, posteriormente al secado se trituró y se realizó la técnica de maceración. Se maceraron $250 \mathrm{~g}$ del material seco en $1000 \mathrm{ml}$ de metanol durante 48 horas a temperatura ambiente y en ausencia de luz. Se obtuvo el extracto líquido de la maceración mediante una filtración con papel filtro (Whatman ® 42) y algodón. El extracto líquido obtenido fue concentrado a presión reducida en un evaporador rotatorio, con el fin de eliminar los solventes y concentrar los metabolitos secundarios, de acuerdo con la metodología descrita por Rivero et al., 2016.

\section{Caracterización química del extracto metanólico de Salix babylonica}

Perfil químico cualitativo: Al extracto se le realizó el perfil químico de acuerdo con el procedimiento descrito por Bañuelos-Valenzuela et al., 2018, para la de determinación de instauraciones, fenolicos, esteroles, triterpenos, cumarinas, sesquiterpenlactonas, flavonoides, alcaloides, tanidos, fluorataninos, esteroides y saponinas.

Cromatografía de gases: La composición química se determinó mediante una cromatografía de gases (CG; Agilent Tecnologías serie $6890 \mathrm{~N}$ fabricado en U.S.A), con una columna polar DB_WAXetr, a $250^{\circ} \mathrm{C}$ y 12.13 psi con un flujo de He $36.5 \mathrm{ml}$ min-1 después de la inyección. Las condiciones para la columna fueron: temperatura inicial 50 ${ }^{\circ} \mathrm{C}$ de cero a dos min, aumentando de 10 en $10^{\circ} \mathrm{C}$ hasta llegar a $250{ }^{\circ} \mathrm{C}$, manteniendo la temperatura constante por 5 min para luego descender a $50^{\circ} \mathrm{C}$ por dos min con un flujo de He de $1.6 \mathrm{ml}$ min-1 a una presión de 12.13 psi y una velocidad promedio de $25 \mathrm{~cm} \mathrm{~s}$ 1 , utilizando un detector de flama ionizante (FID), a una temperatura de $210^{\circ} \mathrm{C}$ con un flujo de $\mathrm{H}_{2}$ de $40 \mathrm{ml}$ min-1 y un flujo de aire de $450 \mathrm{ml}$ min-1. Los estándares (SigmaAldrich), se utilizaron en concentraciones diferentes (cuadro1).

\section{Actividad antibacteriana}

Para determinar la actividad antibacteriana del extracto metanólico de Salíx babylonica, se utilizaron los siguientes métodos: Concentración Mínima Inhibitoria (CMI) y la concentración Mínima Bactericida (CMB), siguiendo las especificaciones del CLSI (CLSI, 2012). 
Cuadro 1. Concentraciones de estándares empleados para la determinación química del extracto metanólico de Salix babylonica mediante cromatografía de gases.

\begin{tabular}{llllll}
\hline \multirow{2}{*}{ Estándar } & \multicolumn{5}{c}{ Compuesto $\mathbf{~ m g} / \mathbf{m L}^{-1}$} \\
\cline { 2 - 6 } & Timol & Carvacrol & Linalol & Terpineno & Limoneno \\
\hline 1 & 10.373 & 8.284 & 7.744 & 7.154 & 8.496 \\
2 & 5.186 & 4.142 & 3.872 & 3.577 & 4.248 \\
3 & 2.593 & 2.071 & 1.936 & 1.789 & 2.124 \\
4 & 1.297 & 1.035 & 0.968 & 0.894 & 1.062 \\
5 & 0.648 & 0.518 & 0.484 & 0.447 & 0.531 \\
6 & 0.324 & 0.259 & 0.242 & 0.224 & 0.265 \\
\hline
\end{tabular}

La prueba de actividad antimicrobiana se llevó a cabo con las cepas ATCC 6538 de Staphylococcus aureus, 6633 de Bacillus subtilis, 35218 de Escherichia coli, 9027 de Pseudomonas aeruginosa, 14028 de Salmonella typhi, 10708 de Salmonella cholerasuis y 19113 de Listeria monocytogenes. Se inoculó una colonia de cada bacteria en caldo nutritivo (BD Bioxon), el cual fue incubado en agitación constante (70 rpm) por 24 horas a 37ํㅡ. Trascurrido el tiempo de incubación, el inóculo se ajustó con caldo nutritivo al 0.5 del patrón de turbidez de Mc Farland, el cual corresponde a 150 × $10^{6} \mathrm{cel} / \mathrm{ml}$.

Para la determinación de la CMI se utilizó el método de microdilución en placa, utilizando concentraciones de 400, 200 100, 50, 25, 12.50, 6.25, $3.12 \mathrm{mg} / \mathrm{ml}$, del extracto metanólico de Salix babylonica. Cada concentración fue preparada con caldo nutritivo (BD Bioxon). El procedimiento se realizó por triplicado en placas de 96 pozos, colocando $100 \mu \mathrm{l}$ de cada una de las diluciones del extracto más $10 \mu \mathrm{l}$ de la suspensión bacteriana, previamente ajustada a 0.5 de McFarland. Una vez realizada la inoculación la placa se incubó a $37^{\circ} \mathrm{C}$ durante 24 horas a $70 \mathrm{rpm}$ en agitación constante, el control positivo fue Kanamicina (AppliChem 4K10421) a concentraciones de 64, 32, 16, 8.0, 4.0, 2.0, 1.0 y $0.5 \mu \mathrm{g} / \mathrm{ml}$ y el control negativo fue caldo nutritivo.

Para determinar la CMI se empleó un método colorimétrico, basado en el uso de sales de tetrazolium (Balouiri et al., 2016). Una vez transcurrido el tiempo de incubación se agregaron $20 \mu \mathrm{l}$ de una solución al $0.04 \%$ (w/v) de p-iodonitrotetrazolium en cada pozo; se incubó por 30 minutos a $37^{\circ} \mathrm{C}$ y se procedió a hacer la lectura, determinándose como la concentración mínima inhibitoria, la concentración a la cual la solución vira a rosa (Kaewpiboon et al., 2012; Mothana et al., 2009).

Para determinar la CMB, previa adición del p-iodonitrotetrazolium, se inocularon $5 \mu \mathrm{l}$ de cada pozo en agar Mueller Hinton, para posteriormente incubar a $37^{\circ} \mathrm{C}$ durante 24 horas. Trascurrido el tiempo de incubación se procedió a hacer la lectura para determinar la concentración mínima bactericida del extracto, es decir la concentración a la cual no se observó crecimiento bacteriano en la placa. 


\section{RESULTADOS}

La caracterización cualitativa realizada al extracto metanólico de Salix babylonica, indican la presencia de insaturaciones, oxidrilos fenólicos, cumarinas, lactonas, flavonoles, quinonas, saponinas, aromaticidad y polifenoles; además de ser positivo a la prueba de Lieberman-Buchard, la cual indica la presencia de triterpenos y compuestos esteroidales. (Cuadro 2).

Cuadro 2. Pruebas cualitativas de perfil químico del extracto metanólico de Salix babylonica.

\begin{tabular}{lcc}
\hline \multicolumn{1}{c}{ Prueba } & Resultado \\
\hline Insaturación & + \\
Oxidrilos Fenolicos & + \\
Cumarinas & + \\
Lactonas & + \\
Salkowski & - \\
Flavonoles & + \\
Flavonas & - \\
Chalconas & - \\
Quinonas & + \\
Shinoda & - \\
Sesquiterpenlactonas & - \\
Agitación & + \\
Bicarbonato & - \\
Saponinas & + \\
Aromaticidad & + \\
Triterpenos & + \\
Taninos & - \\
Florataninos & + \\
Esteroides & + \\
\hline
\end{tabular}

\section{Composición química}

El análisis en el cromatógrafo de gases fue de 20 min con un tiempo de retención para terpineno de $6.40 \mathrm{~min}$, limoneno $6.66 \mathrm{~min}$, linalol $11.28 \mathrm{~min}$, timol $18.04 \mathrm{~min}$ y carvacrol 18.37 min. Para calcular la concentración de las muestras se trabajó con cinco estándares con seis concentraciones cada uno (cuadro 1).

Una vez realizadas las curvas de calibración y teniendo las ecuaciones, se determinó que el extracto metanólico de Salix babylonica contiene Timol y Carvacrol en concentraciones de $0.5319 \mathrm{mg} / \mathrm{ml}, 0.4158 \mathrm{mg} / \mathrm{ml}$ respectivamente. Cuadro 3. 
Cuadro 3. Composición química del extracto metanólico de Salix babylonica

\begin{tabular}{cccccc}
\hline \multirow{2}{*}{$\begin{array}{c}\text { Estándar/ } \\
\text { Extracto }\end{array}$} & Terpineno & Limoneno & Linalol & Timol & Carvacrol \\
\cline { 2 - 6 } & 10.373 & 8.284 & 7.744 & 7.154 & 8.496 \\
\hline 1 & 5.186 & 4.142 & 3.872 & 3.577 & 4.248 \\
2 & 2.593 & 2.071 & 1.936 & 1.789 & 2.124 \\
3 & 1.297 & 1.035 & 0.968 & 0.894 & 1.062 \\
4 & 0.648 & 0.518 & 0.484 & 0.447 & 0.531 \\
5 & 0.324 & 0.259 & 0.242 & 0.224 & 0.265 \\
6 & $\mathbf{0}$ & $\mathbf{0}$ & $\mathbf{0}$ & $\mathbf{0 . 5 3 1 9}$ & $\mathbf{0 . 4 1 5 8}$ \\
\hline Salix & & & & &
\end{tabular}

\section{Actividad antibacteriana}

\section{Concentración mínima inhibitoria}

La concentración mínima inhibitoria del extracto metanólico de Sálix babylonica, fue de $100 \mathrm{mg} / \mathrm{ml}$ para Escherichia coli, Salmonella typhi, Salmonella cholerasuis $y$ Pseudomonas aeruginosa; $25 \mathrm{mg} / \mathrm{ml}$ para Listeria monocytogenes y Staphylococcus aureus. La concentración más baja a la que el extracto tuvo actividad fue de $12.5 \mathrm{mg} / \mathrm{ml}$, frente a Bacillus subtillis (cuadro 4).

Cuadro 4. Concentración Mínima Inhibitoria del extracto metanólico de Salix babylonica

\begin{tabular}{lcccccccc}
\hline \multicolumn{1}{c}{ Bacteria } & \multicolumn{7}{c}{ Concentraciones (mg/mL) } \\
\cline { 2 - 9 } & $\mathbf{4 0 0}$ & $\mathbf{2 0 0}$ & $\mathbf{1 0 0}$ & $\mathbf{5 0}$ & $\mathbf{2 5}$ & $\mathbf{1 2 . 5}$ & $\mathbf{6 . 2 5}$ & $\mathbf{3 . 1 2}$ \\
\hline Escherichia coli & - & - & $\mathrm{CMI}$ & + & + & + & + & + \\
Salmonella typhi & - & - & $\mathrm{CMI}$ & + & + & + & + & + \\
Salmonella cholerasuis & - & - & $\mathrm{CMI}$ & + & + & + & + & + \\
Pseudomonas aeruginosa & - & - & $\mathrm{CMI}$ & + & + & + & + & + \\
Staphylococcus aureus & - & - & - & - & $\mathrm{CMI}$ & + & + & + \\
Listeria monocytogenes & - & - & - & - & $\mathrm{CMI}$ & + & + & + \\
Bacillus subtillis & - & - & - & - & - & $\mathrm{CMI}$ & + & + \\
\hline
\end{tabular}

(-) Sin cambio de color, (+) Cambio de color

\section{Concentración mínima bactericida}

Se determinó que la concentración mínima bactericida del extracto metanólico de Sálix babylonica fue $200 \mathrm{mg} / \mathrm{ml}$; para Escherichia coli, Salmonella typhi, Salmonella cholerasuis y Pseudomonas aeruginosa, $50 \mathrm{mg} / \mathrm{ml}$; y para Listeria monocytogenes y Staphylococcus aureus y de $25 \mathrm{mg} / \mathrm{ml}$ para Bacillus subtillis (cuadro 5). 
Cuadro 5. Concentración Mínima Bactericida del extracto metanólico de Salix babylonica

\begin{tabular}{lcccccccc}
\hline & \multicolumn{7}{c}{ Concentraciones (mg/mL) } \\
\cline { 2 - 9 } \multicolumn{1}{c}{ Bacteria } & $\mathbf{4 0 0}$ & $\mathbf{2 0 0}$ & $\mathbf{1 0 0}$ & $\mathbf{5 0}$ & $\mathbf{2 5}$ & $\mathbf{1 2 . 5}$ & $\mathbf{6 . 2 5}$ & $\mathbf{3 . 1 2}$ \\
\hline Escherichia coli & - & $\mathrm{CMB}$ & + & + & + & + & + & + \\
Salmonella typhi & - & $\mathrm{CMB}$ & + & + & + & + & + & + \\
Salmonella cholerasuis & - & $\mathrm{CMB}$ & + & + & + & + & + & + \\
Pseudomonas aeruginosa & - & $\mathrm{CMB}$ & + & + & + & + & + & + \\
Staphylococcus aureus & - & - & - & $\mathrm{CMB}$ & + & + & + & + \\
Listeria monocytogenes & - & - & - & $\mathrm{CMB}$ & + & + & + & + \\
Bacillus subtillis & - & - & - & - & $\mathrm{CMB}$ & - & & - \\
\hline
\end{tabular}

(-) Sin crecimiento, (+) Crecimiento

\section{DISCUSIÓN}

Por medio de las pruebas cualitativas se determinó la presencia de insaturaciones, oxidrilos fenólicos, cumarinas, lactonas, flavonoles, quinonas, fluorataninos, esteroides triterpenos y saponinas en el extracto metonólico de Salix babylonica. En estudios previos se han identificado compuestos como tritetracontano, 1,2,3-propanetriol éster ácido octadecanoico, éster metílico del ácido hexadecanoico y 1,3-dioxano-4-(hexadecil oxi)-2pentadecilo; la mayoría de ellos clasificados como compuestos fenólicos, además de 7O- $\beta$-D-glucopiranosido de luteolina, luteolina y crisoeriol; compuestos clasificados como flavonoides (Salem et al., 2011).

Se han reportado actividades biológicas como anticancerígena, antiulcerosa, antimalaria, antidiarreica, antimicótica, antitusígena, antinflamatoria, antihelmíntica y antibacteriana; en estudios realizados con compuestos fenólicos, alcaloides, glicósidos y terpenos (Hernández-Alvarado et al., 2018).

Por otro lado, la cromatografía de gases permitió identificar Timol y Carvacrol a concentraciones de $0.5319 \mathrm{mg} / \mathrm{ml}, 0.4158 \mathrm{mg} / \mathrm{ml}$ respectivamente. Estos compuestos están clasificados como aceites esenciales de naturaleza volátil, con algunas actividades biológicas reportadas como: expectorantes, antifúngicas, antiinflamatorias, analgésicas, antisépticas, antioxidantes, antirreumáticas, antiespasmódico, anti-hepatotoxicas y antibacterianas; tanto frente a bacterias Gram positivas como Gram negativas (Magi et al., 2015).

Al realizar la evaluación antibacteriana del extracto metanólico de Salix babylonica, se determinó que el extracto presenta mejor actividad frente a bacterias Gram positivas (Staphylococcus aureus, Listeria monocytogenes y Bacillus subtilis); que contra bacterias Gram negativas (Escherichia coli, Salmonella typhi, Salmonella cholerasuis, Pseudomonas aeruginosa). Este efecto se fundamenta en la estructura propia de las bacterias Gram negativas, que al contar con una membrana fosfolipídica que impide que 
la pared celular sea penetrada por solutos lipofílicos; mientras las porinas constituyen una barrera selectiva para los solutos hidrofílicos, por lo que la bacteria está protegida de ser penetrada por compuestos como antibióticos o algunos metabolitos secundarios derivados de las plantas (Kaye et al., 2004; Ndhlala et al., 2015).

Para la determinacion de la actividad antibacteriana, es importante determinar Concentracion Minima Inhibitoria (CMI) y la Concentracion Minima Bactericida (CMB); definiéndose como $\mathrm{CMl}$ a la concentración más baja de agente antimicrobiano que inhibe el crecimiento del microorganismo, detectados de forma visual (CLSI, 2012). En el presente experimento para determinar el punto final de la $\mathrm{CMI}$, se empleó un método colorimétrico, basado en el uso de sales de tetrazolium (Balouiri et al., 2016); el cual permite observar un cambio de color de amarillo a rosa, provocado por la entrada de esta sal en la célula, la cual se reduce por las oxidorreductasas dependientes de $\mathrm{NAD}(\mathrm{P}) \mathrm{H}$ y las deshidrogenasas de las células metabólicamente activas, produciendo el cambio de color a rosa (Berridge et al., 2005).

Sulaiman et al., en 2013, realizaron un estudio en el cual evaluaron la actividad antimicrobiana del extracto etanólico de corteza de Sálix alba, perteneciente al género sálix y familia salicaeae; igual que Sálix babylonica. En dicho estudio determinaron que Sálix alba tiene mejor actividad antibacteriana contra Staphylococcus aureus; mediana actividad contra Pseudomonas aeruginosa y no presentó efecto contra Escherichia coli y Klebsiella pneumoniae. Las concentraciones evaluadas fueron 10, 20, 40, 60 y $80 \mathrm{mg} / \mathrm{ml}$, utilizando la técnica de difusión en agar; observándose los mayores halos de inhibición a $80 \mathrm{mg} / \mathrm{ml}$. Los resultados de dicho estudio correponden con los observados en el presente experimento, ya que el extracto tuvo mejor efecto contra las bacterias Gram positivas (Staphylococcus aureus, $25 \mathrm{mg} / \mathrm{ml}$; Listeria monocytogenes, $25 \mathrm{mg} / \mathrm{ml}$ y Bacillus subtilis, $12.5 \mathrm{mg} / \mathrm{ml}$ ), que contra las bacterias Gram negativas (Escherichia coli, Salmonella typhi, Salmonella cholerasuis y Pseudomonas aeruginosa, $100 \mathrm{mg} / \mathrm{ml}$, para cada una); sin embargo, el extracto se obtuvo de la corteza de Sálix alba. Las concentraciones cambian y la técnica también, por lo que los resultados no son $100 \%$ comparables, aunque las arbóreas pertenecen al mismo género y familia.

Por otro lado, en un estudio realizado por Wahab y colaboradores en 2018, quienes evaluaron los extractos metanólicos de las hojas y la corteza de Sálix babylonica; además de sus fracciones de éter de petróleo, cloruro de metileno y acetato de etilo (diluidos en dimetilsulfóxido), para determinar su actividad antimicrobiana frente a bacterias Gram positivas (Staphylococcus aureus) y Gram negativas (Escherichia coli, Klebsiella pneumoniae y Pseudomonas aeruginosa), utilizando la técnica de difusión en agar y el extracto a una concentracion de $100 \mu \mathrm{g}$. Los resultados mostraron que tanto el extracto metanólico de las hojas y de la corteza tienen actividad antimicrobiana de moderada o débil, contra los microorganismos desafiados; observándose los mayores halos de 
inhibición (10 mm) con Pseudomonas aeruginosa, seguida de Klebsiella pneumoniae (9 $\mathrm{mm}$ ), finalmente Escherichia coli, Staphylococcus aureus (8 mm).

Debido a que en los estudios mencionados únicamente se aplican técnicas para determinar la sensibilidad del microorganismo a determinado compuesto por el método de difusión en agar; no es posible comparar los resultados con los obtenidos en el presente experimento, ya que en el presente se determinó la concentración mínima inhibitoria por el método de microdilución en placa; además de que en el estudio de Wahab y colaboradores, se utilizó dimetil sulfóxido, para diluir los extractos y fracciones; compuesto que se utiliza para incrementar la permeabilidad de la membrana bacteriana, incrementando la actividad de los compuestos y reduciendo las concentraciones de uso (Borges et al., 2013; Sulaiman et al., 2013; Wahab et al., 2018).

La Concentracion Minima Inhibitoria, se define como la concentración más baja de agente antimicrobiano-necesaria para matar el 99,9\% del inóculo final, después de la incubación durante $24 \mathrm{~h}$ bajo un conjunto estandarizado de condiciones descrito por el CLSI (Balouiri et al., 2016). La determinacion de la CMI no es una opción viable para conocer al $100 \%$ la eficacia de un fármaco o compuesto, ya que dentro de cada pozo todavía puede haber células viables si el fármaco evaluado solo tuvo un efecto bacteriostático sobre las especies bacterianas en estudio (Wiegand et al., 2008).

En el presente experimento se determiaron las CMB del extracto metanólico, frente a Escherichia coli, Salmonella typhi, Salmonella cholerasuis y Pseudomonas aeruginosa (200 mg/ml); Listeria monocytogenes y Staphylococcus aureus (50 mg/ml) y Bacillus subtillis (25 mg/ml); sin embargo, no existen estudios reportados con Sálix babylonica u otra especie del género Sálix, con el cual se haya reportado dicha actividad.

\section{CONCLUSIÓN}

En el presente estudio se demostró que el extracto metanólico de Sálix babylonica tiene actividad antibacteriana potencial sobre algunos patógenos bacterianos de importancia en salud pública; siendo una alternativa para el tratamiento de enfermedades producidas por bacterias resistentes o multirresistentes a antibióticos.

\section{LITERATURA CITADA}

ALÓS Jl. 2014. Resistencia bacteriana a los antibóticos: una crisis global. Enfermedades Infecciosas

y Microbiologias

Clinica. 33(10):692-699. http://dx.doi.org/10.1016/j.eimc.2014.10.004

BALOUIRI M, Sadiki M, Ibnsouda SK. 2016. Methods for in vitro evaluating antimicrobial activity: A review. Journal of Pharmaceutical Analysis. 6(2):71-79. https://doi.org/10.1016/j.jpha.2015.11.005. 
BAÑUELOS-VALENZUELA R, Delgadillo L, Chairez F, Delgadillo O, Meza-López C. 2018. Composición química y FTIR de extractos etanólicos de Larrea tridentata, Origanum vulgare, Artemisa ludoviciana y Ruta graveolens Agrociencia. 52(3): 309-321. https://dialnet.unirioja.es/servlet/articulo?codigo=6423180.

BERRIDGE MV, Herst PM, Tan AS. 2005. Tetrazolium dyes as tools in cell biology: new insights into their cellular reduction. Biotechnology Annual Review. 11:127-152. https://doi.org/10.1016/s1387-2656(05)11004-7.

BORGES A, Ferreira C, Saavedra MJ, Simoes, M. 2013. Antibacterial activity and mode of action of ferulic and gallic acids against pathogenic bacteria. Microbial Drug Resistance. 19(4): 256-265. https://doi.org/10.1089/mdr.2012.0244.

CLSI (Clinical and Laboratory Standards Institute). 2012. Methods for Dilution Antimicrobial Susceptibility Tests for Bacteria That Grow Aerobically; Approved StandardNinth Edition. Pp. 88. USA.

HERNÁNDEZ-ALVARADO J, Zaragoza-Bastida A, López-Rodríguez G, Peláez-Acero A, Olmedo-Juárez A, Rivero-Perez N. 2018. Actividad antibacteriana y sobre nematodos gastrointestinales de metabolitos secundarios vegetales: enfoque en Medicina Veterinaria. Abanico Veterinario. 8(1):14-27. http://dx.doi.org/10.21929/abavet2018.81.1.

KAEWPIBOON C, Lirdprapamongkol K, Srisomsap C, Winayanuwattikun P, Yongvanich T, Puwaprisirisan P, Svasti J, Assavalapsakul W. 2012. Studies of the in vitro cytotoxic, antioxidant, lipase inhibitory and antimicrobial activities of selected Thai medicinal plants. BMC Complementary and Alternative Medicine. 12(1):217. https://doi.org/10.1186/14726882-12-217.

KAYE KS, Engemann JJ, Fraimow HS, Abrutyn E. 2004. Pathogens resistant to antimicrobial agents: epidemiology, molecular mechanisms, and clinical management. Infectious disease clinics of North America. 18(3):467-511. https://doi.org/10.1016/j.idc.2004.04.003.

KHAN UA, Rahman H, Niaz Z, Qasim M, Khan J, Tayyaba, Rehman B. 2013. Antibacterial activity of some medicinal plants against selected human pathogenic bacteria. European Journal of Microbiology and Immunology. 3(4): 272274. https://doi.org/10.1556/EuJMI.3.2013.4.6

LOZANO R, Naghavi M, Foreman K, Lim S, Shibuya K, Aboyans V, et al., 2012.Global and regional mortality from 235 causes of death for 20 age groups in 1990 and 2010: a systematic analysis for the Global Burden of Disease Study 2010. 2012. The Lancet. 380(9859):2095-2128. https://doi.org/10.1016/S0140-6736(12)61728-0

MAGI G, Marini E, Facinelli B. 2015. Antimicrobial activity of essential oils and carvacrol, and synergy of carvacrol and erythromycin, against clinical, erythromycin-resistant Group 
$\begin{array}{llll}\text { A Streptococci. } & \text { Frontiers in }\end{array}$ https://doi.org/10.3389/fmicb.2015.00165.

MOTHANA RA, Lindequist U, Gruenert R, Bednarski PJ. 2009. Studies of the in vitro anticancer, antimicrobial and antioxidant potentials of selected Yemeni medicinal plants from the island Soqotra. BMC Complementary and Alternative Medicine. 9: 7. https://doi.org/10.1186/1472-6882-9-7.

NDHLALA AR, Ghebrehiwot HM, Ncube B, Aremu AOJ, Gruz M, Subrtova J, Van Staden A. 2015. Antimicrobial, anthelmintic activities and characterization of functional phenolic acids of Achyranthes aspera linn, a medicinal plant used for the treatment of wounds and ringworm in east Africa. Frontiers in Pharmacology. 6:274. https://doi.org/10.3389/fphar.2015.00274.

RENISHEYA JJMT, Johnson M, Mary UM, Arthy A. 2011. Antibacterial activity of ethanolic extracts of selected medicinal plants against human pathogens. Asian Pacific Journal of Tropical Biomedicine.1(1):S76-S78. https://doi.org/10.1016/S2221-1691(11)60128-7.

RIVERO-PEREZ N, Ayala-Martínez M, Zepeda-Bastida A, Meneses-Mayo M, OjedaRamírez D. 2016. Anti-inflammatory effect of aqueous extracts of spent Pleurotus ostreatus substrates in mouse ears treated with 12-O-tetradecanoylphorbol-13-acetate. Indian Journal of Pharmacology. 48(2):141-144. https://dx.doi.org/10.4103\%2F02537613.178826.

SALEM AFZ, Salem MZ, González-Ronquillo M, Camacho LM, Cipriano M. 2011. Major chemical constituents of Leucaena leucocephala and Salix babylonica leaf extracts. Journal of Tropical Agriculture. 49: 95-98. http://jtropag.kau.in/index.php/ojs2/article/view/244

SULAIMAN GM, Hussien NN, Marzoog TR, Awad, HA. 2013. Phenolic content, antioxidant, antimicrobial and cytotoxic activities of ethanolic extract of Salix alba. American Journal of Biochemistry and Biotechnology. 9(1): 41-46. https://thescipub.com/PDF/ajbbsp.2013.41.46.pdf.

WAHAB GA, Sallam A, Elgaml A, Lahhloub M, Afifi MS. 2018. Antioxidant and antimicrobial activities of Salix babylonica extracts. World Journal of Pharmaceutical Sciences. 6(4): 1-6. http://www.wjpsonline.org/.

WHO (World Health Organization). 2017. Antimicrobial resistance. https://www.who.int/antimicrobial-resistance/en/

WIEGAND I, Hilpert K, Hancock REW. 2008. Agar and broth dilution methods to determine the minimal inhibitory concentration (MIC) of antimicrobial substances. Nature Protocols. 3(2):163-175. https://doi.org/10.1038/nprot.2007.521. 